Christiane Bayerl ${ }^{1}$
M. Kurzen ${ }^{1}$
I. Treiss $^{2}$
I. Haußer
T. Walker

\section{Sjögren-Larsson-Syndrom mit reduzierter Fettaldehyddehydrogenase-Aktivität bei einem Kollodiumbaby und konsanguinen Eltern}

\author{
Sjoegren-Larsson Syndrome and Reduced Fatty Aldehyde Dehydrogenase \\ in a Collodium Baby and Consanguine Parents
}

\section{Zusammenfassung}

Das Sjögren-Larsson-Syndrom (SLS) ist ein seltenes autosomal rezessiv vererbtes Syndrom mit seit Geburt bestehender Ichthyosis, spastischer Di-/Tetraplegie, geistiger Retardierung und kompletter oder inkompletter Reduktion der Fettaldehyddehydrogenase (FALDH)-Aktivität, deren Gen auf Chromosom 17p11.2 lokalisiert ist. Wir sahen drei Tage nach Geburt ein Kollodiumbaby konsanguiner Eltern konsiliarisch mit Erythrodermie, lamellöser Schuppung und Hyperkeratose an Handflächen und Fußsohlen. Mit 3,5 Jahren trat eine Spastik an der unteren Extremität auf, mentale Entwicklungsstörung, Hyperkeratose und Schuppung in der Nackenregion, am Rumpf und an den Beugen. Eine Biopsie im 8. Lebensjahr zeigte lichtmikroskopisch eine Akanthose, ein schmales Stratum granulosum und eine Hyperkeratose mit wabig geblähten Bereichen. Elektronenmikroskopisch zeigte sich ein unauffälliges Stratum spinosum, unauffällige Hyalingranula im Stratum granulosum, eine teilweise irreguläre Ausschleusung von Keratinosomen und in den Hornlamellen, keine Lipidtropfen, keine Cholesterinspalten und keine charakteristischen Membranstapel. In der enzymatischen Untersuchung an Fibroblastenkulturen des Jungen konnte eine Reduktion der FALDH-Aktivität nachgewiesen werden. Die Mutationsanalyse in Lymphozyten zeigte homozygote Komplexmutationen mit einer Transversion $901 \mathrm{G}$ nach $\mathrm{C}$ und eine Nukleotid-Deletion 906delT. Die gleiche Komplexmutation wurde heterozygot bei Vater und Mutter gefunden. Dies ist der zweite Bericht über das seltene Ereignis einer Komplexmutation in der FALDH cDNA bei SLS.

\section{Abstract}

Sjoegren-Larsson Syndrome (SLS) is a rare autosomal recessive disorder characterized by ichthyosis after birth, spastic di- or tetraplegia, mental retardation and complete or incomplete reduction in fatty aldehyde dehydrogenase (FALDH) activity, the gene localized on chromosome 17p11.2. Three days after birth we saw a collodium baby of consanguine parents with erythrodermia and hyperkeratosis of palms and soles. In the age of 3,5 the boy developed a spastic of the leg, mental retardation, hyperkeratosis and desquamation in the neck, flank and flexural parts of the body. A biopsy at the age of 8 years revealed acanthosis, a reduced stratum granulosum and a hyperkeratotic puffed up stratum corneum. Electromicroscopy showed an intact stratum spinosum, intact hyaline granules in stratum granulosum, a partially irregular drop out of keratinosomes, no lipid droplets, no cholesterin gaps and no membrane staples. Enzymatic analysis of fibroblast cultures from the boy resulted in reduced FALDHactivity. Mutation analysis in lymphocytes showed homozygous complex mutations with the transversion $901 \mathrm{G} \rightarrow C$ and a nucleotide deletion 906delT. The same complex mutation was found in the heterozygote state in father and mother. This is the second report about this complex mutation in the FALDH cDNA in SLS. 
Mehr als 700 Genodermatosen wurden bisher vor allem nach klinischen Gesichtspunkten diagnostiziert. Die Zahl neu entdeckter Genloci für Genodermatosen nimmt stetig zu. Im McKusick-Katalog findet sich die Auflistung der genetischen Erkrankungen. Mehr als ein Drittel der Genodermatosen sind mittlerweile kartiert [6]. Als Beispiel für die moderne Diagnostik, unterstützt durch die molekularbiologische Analyse, wird die Geschichte einer Familie mit Sjögren-Larsson-Syndrom beim erstgeborenen Sohn und weiterem Kinderwunsch der Familie vorgestellt.

Die Trias der kongenitalen Ichthyosis, der bis zur Pubertät progredienten Para- oder Tetraspastik und die mentale Retardierung kennzeichnen das Sjögren-Larsson-Syndrom als seltene autosomal rezessive Erbkrankheit [13]. Assoziiert finden sich häufiger zerebrale Krampfanfälle, Kleinwüchsigkeit, „glistening white dots" in der Makula als Korrelat der fettigen Degeneration der retinalen Mikroglia, Netzhautdegeneration, Blepharitis, Keratitis und seltener Kyphose der Brustwirbelsäule, Hypertelorismus, weite Interdentalräume, Schmelzhypoplasie und Metaphysendysplasie $[2,7]$.

Berichte über das Krankheitsbild stammen vor allem aus europäischen Ländern, aber auch von anderen Kontinenten, jedoch wird das gehäufte Auftreten in Schweden im Ort Västerbotten und Nordbayern mit dem Dreißigjährigen Krieg in Zusammenhang gebracht [2]. Diese geographisch-historische Hypothese basiert auf den Feldzügen Gustav II. Adolfs von Schweden. 1632-1635 waren in Nürnberg und Fürth Lager der schwedischen Armee, in denen auch die protestantische Bevölkerung aus dem Nürnberger Raum Zuflucht fand, so dass unterschiedliche ethnische Gruppen jahrelang zusammenlebten.

Dem SLS liegt biochemisch eine verminderte Aktivität der Fettalkoholdehydrogenase (FALDH) zugrunde [9]. Dieses mikrosomale nicotinamide-adenine-dinucleotid abhängige Enzym ist notwendig für die Oxidation langkettiger aliphatischer Aldehyde in Fettsäuren. FALDH ist eine Komponente des Fettalkohol:NAD ${ }^{+}$ Oxidoreduktase-Komplexes, der die Oxidation der Fettalkohole katalysiert. Die Ansammlung von Fettalkoholen, Fettaldehyden und dazugehöriger Lipid-Metaboliten wird als Ursache für die klinische Symptomatik angesehen [11]. Das Gen der FALDH (Synonym „ALDH10“) wurde molekulargenetisch auf Chromosom 17 in Region p11.2 lokalisiert und ist sequenziert [3].

\section{Kasuistik}

\section{Anamnese}

27-jährige Erstgebärende, erste Schwangerschaft, Spontangeburt eines Jungen aus Schädellage nach vorzeitigem Blasensprung in der 36. SSW, Nabelschnurumschlingung einmal um den Hals, Apgar 7/9/10, Geburtsgewicht 2570 g, Länge $48 \mathrm{~cm}$, Kopfumfang $29 \mathrm{~cm}$, Kollodiumbaby. Lunge, Cor und neurologische Untersuchung unauffällig, Augenhintergrund regelrecht. Neugeborenenschnellteste unauffällig. Bilirubin mit $18,2 \mathrm{mg} \%$ am 5 . Lebenstag erhöht. Familienanamnestisch türkische konsanguine Eltern (Cousin/Cousine I. Grades), keine Erkrankungen bekannt. Mit 4 Jahren Vorstellung in der neuropädiatrischen Ambulanz mit psychomotorischer Entwicklungsverzögerung, besonders Sprachentwicklungsverzögerung bei gutem Sprachverständnis. Ataktisches Gangbild mit breitbeinigem Laufen ohne Abrollen mit er-

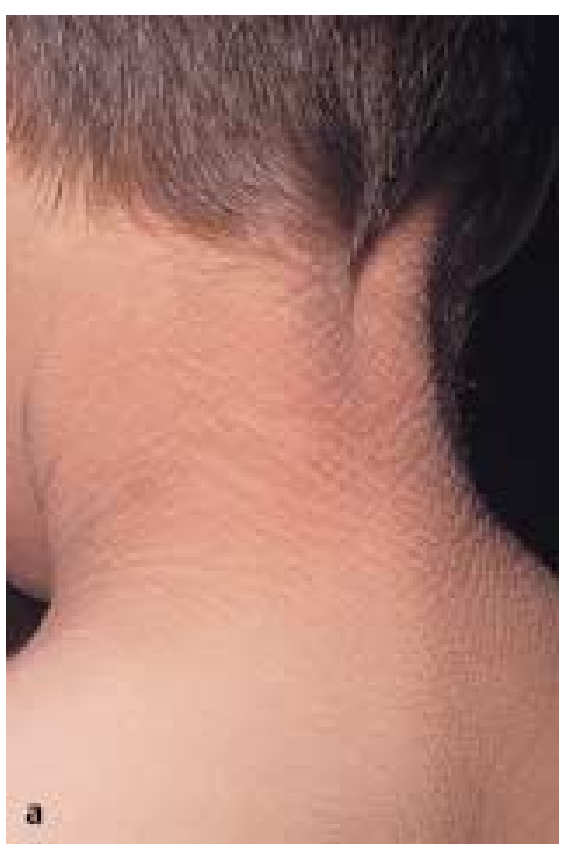

Abb. 1 Gelbliche bis dunkelbraune papillomatöse Keratosen, die der Haut ein lichenifiziertes Bild verleihen, $\mathbf{a}$ in der Nackenpartie und $\mathbf{b}$ am Rumpf des 8-jährigen Jungen mit SLS.

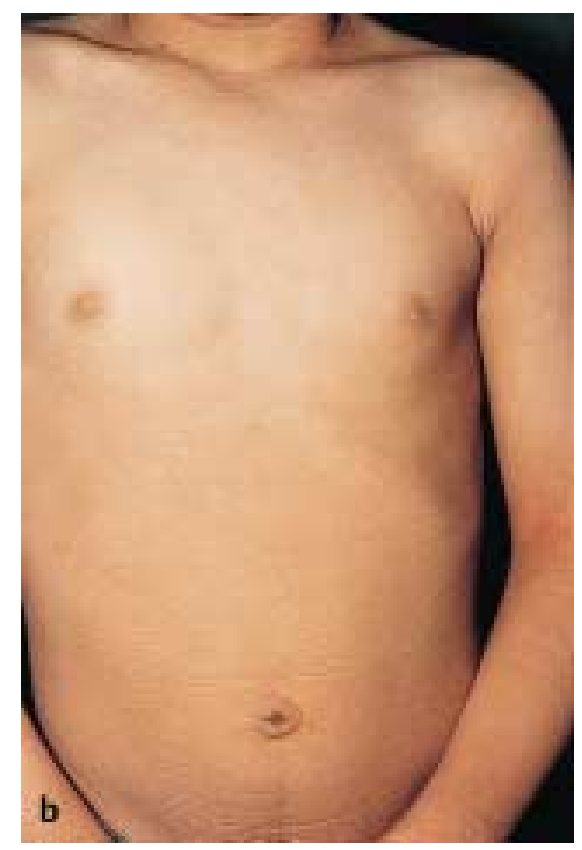

hobenen Armen. Muskeleigenreflexe seitengleich lebhaft und grobe Kraft normal bis mäßig vermindert. Seit einem Jahr Krankengymnastik. Einleitung einer Ergotherapie. Der V.a. SLS bestand aufgrund des Verlaufs und klinischen Bildes, bisher hatten die Eltern jedoch eine weitere Abklärung abgelehnt. Grund der erneuten Vorstellung des Jungen mit seinen Eltern war der aktuelle Kinderwunsch des Paares.

\section{Hautbefund}

2 Stunden nach Geburt panzerartige Haut, vor allem an Handflächen und Fußsohlen mit Rhagaden, im Gesicht ebenfalls verdickte Haut, gerötete Augenlider, keine Wimpern, hochroter spitzer Mund. Haut am Körper pergamentartig, Hände geschwollen, Finger dadurch fast kontrakt. 3 Tage nach der Geburt Erythrodermie, Ichthyosis an Beugen, Hyperkeratose an Handflächen und Fußsohlen, Kontraktur der Hände und Fußsohlen. Mit 4 Jahren sehr trockene, gerötete, hyperkeratotische, lichenifiziert erscheinen- 
de Haut, vor allem im Nacken und auf den Handrücken. Mit 8 Jahren generalisierte Ichthyose (Abb.1 a u. b), kein Juckreiz, keine Exkoriationen; weit auseinander stehende Zähne mit Schmelzdefekten (Abb. 2).

\section{Pathomorphologie der Haut}

Lichtmikroskopisch akanthotische Epidermis, schmales Stratum granulosum, hyperkeratotische Hornschicht, teils mit dicht übereinander liegenden Hornlamellen, teils breite, lockere und wabig geblähte Hornschicht. Mildes perivaskuläres Infiltrat in der papillären Dermis.

Elektronenmikroskopisch Stratum spinosum unauffällig, Stratum granulosum mit 4 Zelllagen und unauffälligen Keratohyalingranula. Teils normale Keratinosomen, teils defekte Keratinosomen, teils irreguläre Ausschleusung von Keratinosomen in einer Art „Stau“ an der jeweils apikalen Plasmamembran der Keratinosomen. Transitzellen an der Grenze zur Hornschicht. In den Hornlamellen keine Lipidtropfen, keine Cholesterinspalten, keine Membranstapel. „Unruhe“ im Keratinmuster, aber keine Parakeratose. Melaningranula in den Hornlamellen (Dr. rer. nat. I. Haußer, Elektronenmikroskopisches Labor, Hautklinik der Universitätsklink Heidelberg).

\section{Diagnostik}

Mit 4 Jahren Chromosomenanalyse mit normalem unauffälligen Chromosomensatz $46 \mathrm{XY}$, kein Hinweis auf fragiles X-Syndrom. Mukopolysaccharidausscheidung im Urin und lysosomale Enzyme unauffällig. Urinscreening auf organische Säuren und Aminosäuren unauffällig. Kernspintomographie: Signalanhebung des paraventrikulären Marklagers im Sinne einer Leukodystrophie, möglicherweise im Rahmen einer peripartalen Asphyxie, kein Hinweis für eine Missbildung des ZNS. Schlaf-EEG unauffällig.

Mit 8 Jahren Bestimmung der Fettaldehyde in Fibroblastenkultur: FALDH-Aktivität deutlich erniedrigt. Befund vereinbar mit SLS.

Molekulargenetische Untersuchungen, Mutationsanalyse des FALDH-Genes in Lymphozyten (Dr. H. R. Waterham, Emma Children's Hospital AMC, University of Amsterdam, Laboratory of Genetic Metabolic Diseases): Für den 8-jährigen Sohn: homozygot

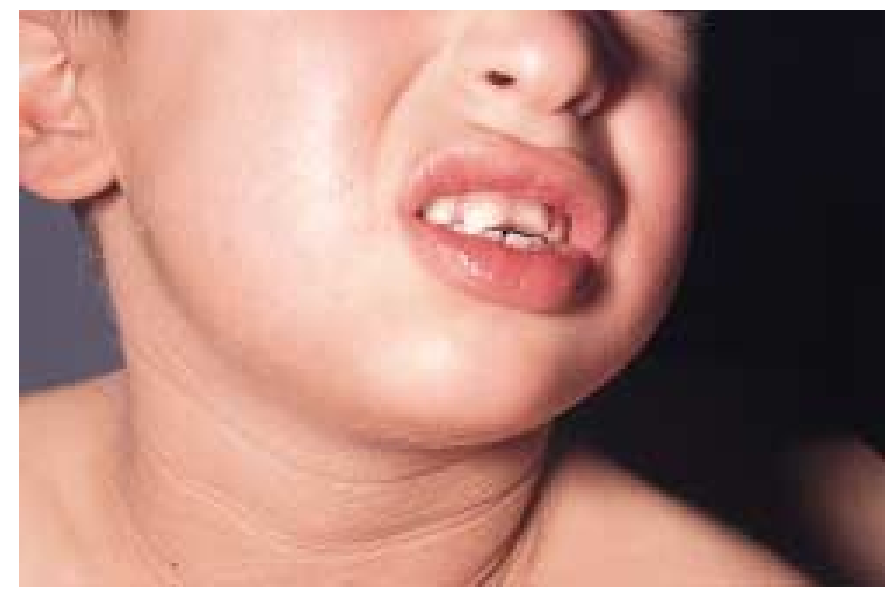

Abb. 2 Kein obligates Zeichen des SLS, aber mitunter assoziiert, ist der weite Abstand der Zähne.

für Mutation $901 \mathrm{G} \rightarrow \mathrm{C}$ und 906delT (selbes Allel), für Vater und Mutter jeweils heterozygot für Mutation $901 \mathrm{G} \rightarrow \mathrm{C}$ und 906delT. Molekulargenetische Bestätigung der Diagnose SLS.

\section{Verlauf}

Lokal harnstoffhaltige Externa ausreichend, da kein Juckreiz, weiter Ergotherapie und Krankengymnastik.

\section{Diskussion}

Das Sjögren-Larsson-Syndrom präsentiert keine spezifischen oder charakteristischen histologischen oder ultrastrukturellen Veränderungen in der Epidermis. Jedoch konnten die entsprechend charakteristischen Ichthyosen bzw. Verhornungsstörungen wie die autosomal-dominante Ichthyosis vulgaris, eine bullöse Erythrodermie und die Ichthyosis congenita der Typen I- IV ausgeschlossen werden [14]. Das Tay-(PIBIDS) Syndrom entfällt, da die Haare unseres Patienten unauffällig waren. Polarisationmikroskopisch würde sich bei diesem Syndrom ein Tigerschwanzmuster des Haares aufgrund des Schwefelmangels zeigen [8]. Tab. 1 zeigt eine Auswahl weiterer Differenzialdiagnosen.

Tab.1 Differenzialdiagnosen zum SLS (Auswahl)

\begin{tabular}{|c|c|c|c|}
\hline Erkrankung & Haupt-Symptomatik & Erbgang & Genlocus \\
\hline Sjögren-Larsson-Syndrom (FALDH) & $\begin{array}{l}\text { Ichthyosis, spastische Di/Tetraplegie, } \\
\text { geistige Retardierung }\end{array}$ & autosomal rezessiv & 17p11.2 \\
\hline $\begin{array}{l}\text { PIBIDS-Syndrom (Tay-Syndrom) } \\
\text { (XP Gruppe D Mutation) }\end{array}$ & $\begin{array}{l}\text { Photosensitivität, Ichthyosis, } \\
\text { „brittle hair“, Intelligenz-Defizit, } \\
\text { „decreased fertility“, „short stature“ }\end{array}$ & autosomal rezessiv & 19q13.2-q13.3 \\
\hline Ichthyosis congenita & $\begin{array}{l}\text { mit und ohne Erythrodermie, } \\
\text { Ektropium, Palmoplantarkeratosen }\end{array}$ & autosomal rezessiv & $\begin{array}{l}14 q 11.2 \\
2 q 33-q 35 \\
19 p 13.1-q 12 \\
3 p 21\end{array}$ \\
\hline $\begin{array}{l}\text { Ichthyosis vulg., X-chromosomal } \\
\text { rezessiver Typ (Steroidsulfatase) }\end{array}$ & $\begin{array}{l}\text { Rumpf- und Streckseiten follikuläre } \\
\text { Keratosen, Ichthyosishand/fuß }\end{array}$ & X-chromosomal rezessiv & Xp22.32 \\
\hline $\begin{array}{l}\text { Chanarin-Dorfman-Syndrom } \\
\text { (Neutrallipidspeicherkrankheit) }\end{array}$ & $\begin{array}{l}\text { Ichthyosis, Katarakt, Myelopathie, Taubheit, } \\
\text { mentale Retardierung und Wachstumsverzögerung }\end{array}$ & autosomal rezessiv & $3 p 21$ \\
\hline $\begin{array}{l}\text { Refsum-Syndrom } \\
\text { (Phytansäureoxidase) }\end{array}$ & $\begin{array}{l}\text { Ichthyosis, Nachtblindheit, Taubheit, } \\
\text { periphere Neuropathie }\end{array}$ & autosomal rezessiv & 10pter-p11.2 \\
\hline
\end{tabular}


Die Erythrodermie bei SLS hat unterschiedliche Ausprägungen in der Kindheit und ist als typisch anzusehen. Eine Kollodiummembran bei SLS wie bei unserem Patienten wird selten gefunden, von manchen Autoren verneint [14].

Die FALDH-Aktivität bei Patienten mit SLS beträgt bei den homozygoten symptomlosen Erbmalsträgern 10\% der normalen Aktivität, bei heterozygoten $50 \%$. Bei unserem Patienten konnte bei konsanguinen Eltern eine deutliche Reduktion der FALDH-Aktivität nachgewiesen werden mit dem klinisch typischen Bild des Syndroms. Die unterschiedliche Ausprägung der Defizienz der FALDH wird nach biochemischen Studien als Erklärung für die milderen inkompletten Verlaufsformen des SLS herangezogen $[1,4,15]$. Dagegen spricht, dass das Zusammentreffen von Ichthyosis, mentaler Retardierung und Spastizität auch bei normaler FALDH-Aktivität gefunden wird. Dieser Symptomenkomplex wurde daher vom SLS folgerichtig abgetrennt und als neues neurokutanes Syndrom eingeordnet [5].

Das FALDH-Gen liegt auf Chromosom 17p11.2. Bisher wurden verschiedene pathogene Mutationen gefunden [15]. In Europa finden sich vor allem zwei Mutationen, $943 \mathrm{C} \rightarrow \mathrm{T}$ und 1297-1298del GA. Erstaunlich ist jedoch vor allem die genetische Heterogenität des SLS, da sich bei der Analyse von 63 Probanden 49 unterschiedliche Mutationen zeigten, einschließlich Deletionen, Insertionen, Aminosäuresubstitutionen und Nonsense-Mutationen. Nur 3 Komplexmutationen wurden gefunden, wobei unser Ergebnis der Transversion $901 \mathrm{G} \rightarrow C$ und der Nukleotid-Deletion 906delT in Exon 6 Teil einer bereits beschriebenen Komplexmutation bei Europäern ist [11].

Typisch für die autosomal rezessive Erbkrankheit ist, dass die heterozygoten Eltern Anlageträger sind, aber klinisch gesund, für Kinder ein Krankheitsrisiko von $25 \%$ bei diesem autosomal rezessiven Erbgang besteht. Die pränatale Diagnostik kann aus Zellen des Fruchtwassers gestellt werden über Nachweis der Speicherung von Fettaldehyden [2] oder basierend auf der Mutations-Analyse aus kultivierten Amniozyten oder Choriongewebe $[10,12]$. Da bei den Eltern unseres Patienten Kinderwunsch bestand, hatten sie sich entschieden, eine pränatale Diagnostik zum SLS bei der nächsten Schwangerschaft durchführen zu lassen. Aufgrund der Konsanguinität besteht jedoch weiterhin ein erhöhtes Risiko für andere Genodermatosen, über das aufgeklärt wurde.

\section{Literatur}

${ }^{1}$ Aoki N, Suzuki H, Ito K, Ito M. A novel point mutation of the FALDH gene in a japanese family with Sjögren-Larsson Syndrome. J Invest Dermatol 2000; 114: 1065-1066

2 Braun-Quentin C, Bathke KD, Pfeiffer RA. Das Sjögren-Larsson-Syndrom in Deutschland. Deutsches Ärzteblatt 1996; 93: B-1039-1042

${ }^{3}$ de Laurenzi V, Rogers GR, Hamrock DJ et al. Sjögren-Larsson syndrome is caused by mutations in the fatty aldehyde dehydrogenase gene. Nat Genet 1996; 12: $52-57$

${ }^{4}$ Kawakami T, Saito R, Fujikawa Y et al. Incomplete Sjögren-Larsson syndrome in two japanese siblings. Dermatology 1999; 198: 93 - 96

${ }^{5}$ Koone MD, Rizzo WB, Elias PM, Williams ML, Lightner V, Pinell SR. Ichthyosis, mental retardation, and asymptomatic spasticity. A new neurocutaneous syndrome with normal fatty alcohol:NAD ${ }^{+}$oxidoreductase activity. Arch Dermatol 1990; 126: 1485-1490

${ }^{6}$ Küster W, Hennies HC, Happle R. Kartierung und molekulare Analyse erblicher Hautkrankheiten. Hautarzt 2000; 51: 906-914

${ }^{7}$ Möhrenschlager M, Rizzo WB, Kraus CS, Limbrock J, Cohen M, AntonLamprecht I, Abeck D, Ring J. Sjögren-Larsson-Syndrom. Hautarzt 2000; 51: 250-255

${ }^{8}$ Rebora A, Crovoto F. PIBIDS-syndrome - trichothiodystrophy with xeroderma pigmentosum (group D) mutations. J Am Acad Dermatol 1987; 16: 940-947

${ }^{9}$ Rizzo WB, Craft DA. Sjögren-Larsson syndrome: deficient activity of the fatty aldehyde dehydrogenase component of fatty aldehyde dehydrogenase component of fatty alcohol:NAD+ oxidoreductase in cultured fibroblasts. J Clin Invest 1991; 88: 1643 - 1648

${ }^{10}$ Rizzo WB, Craft DA, Kelson TL et al. Prenatal diagnosis of Sjögren-Larsson Syndrome using enzymatic methods. Prenat Diagn 1994; 14: $577-581$

${ }^{11}$ Rizzo WB, Carney G, Lin Z. The molecular basis of Sjögren-Larsson Syndrome: mutation analysis of the fatty aldehyde dehydrogenase gene. Am J Hum Genet 1999; 65: 1547 - 1560

${ }^{12}$ Sillen A, Holmgren G, Wadelius C. First prenatal diagnosis by mutation analysis in a family with Sjögren-Larsson syndrome. Prenat Diagn 1997; 17: $1147-1149$

${ }^{13}$ Sjögren T, Larsson T. Oligophrenia in combination with congenital ichthyosis and spastic disorders. Acta Psychiatr Neurol Scand 1957; 32 Suppl 113: 1- 113

${ }^{14}$ Spraker MK. Differential diagnosis of neonatal erythroderma. Ichthyosis. In: Textbook of pediatric dermatology, Vol 1 Harper J, Oranje A, Prose N (Hrsg). Blackwell Science, 1999: 94-95

${ }^{15}$ Willemsen MA, Ijlst L, Steijlen PM et al. Clinical, biochemical and molecular genetic characteristics of patients with the Sjögren-Larsson syndrome. Brain 2001; 124: 1426-1437 